\title{
Acceptability of endoscopic submucosal dissection for sessile serrated lesions: comparison with non-sessile serrated lesions
}

\section{두)(요우}

\author{
Authors \\ Yuichiro Kuroki, Toshiyuki Endo, Kenta Iwahashi, Naoki Miyao, Reika Suzuki, Kunio Asonuma, Yorimasa Yamamoto, \\ Masatsugu Nagahama
}

Institution

Department of Gastroenterology, Showa University

Fujigaoka Hospital, Kanagawa, Japan

submitted 24.6.2020

accepted after revision 3.9 .2020

\section{Bibliography}

Endoscopy International Open 2020; 08: E1832-E1839

DOI 10.1055/a-1268-7353

ISSN 2364-3722

(C) 2020. The Author(s).

This is an open access article published by Thieme under the terms of the Creative Commons Attribution-NonDerivative-NonCommercial License, permitting copying and reproduction so long as the original work is given appropriate credit. Contents may not be used for commecial purposes, or adapted, remixed, transformed or built upon. (https://creativecommons.org/licenses/by-nc-nd/4.0/)

\section{Corresponding author}

Yuichiro Kuroki MD, PhD, Department of Gastroenterology, Showa University Fujigaoka Hospital, 1-30 Fujigaoka, Aobaku, Yokohama 227-8501, Japan

Fax: +81-45-971-3824

yu-kuroki@med.showa-u.ac.jp

\section{ABSTRACT}

Background and study aims Sessile serrated lesions (SSL) are major precursor lesions of serrated pathway cancers, and appropriate treatment may prevent interval colorectal cancer. Studies have reported the outcomes of endoscopic mucosal resection (EMR) for SSL; however, there are insufficient reports on endoscopic submucosal dissection (ESD). We examined the characteristics and outcomes of SSL and compared them to those of non-SSL in ESD.

Patients and methods We reviewed 370 consecutive cases in 322 patients who underwent colorectal ESD between January 2016 and March 2020 at our hospital. There were 2670 -Ila lesions that were stratified into $41 \mathrm{SSL}$ and 226 non-SSL (intramucosal cancer, adenoma) cases. We used propensity matching to adjust for the variances in the factors affecting treatment between the SSL and non-SSL groups.

Results In the baseline cases, young women and proximal colon tumor location were significantly more common in the SSL group. There were no statistically significant differences between the SSL and non-SSL groups in terms of en bloc resection rate ( $97.6 \%$ vs. $99.6 \% ; P=0.28)$, R0 resection rate $(92.7 \%$ vs. $93.4 \% ; P=0.74$ ), perforation ( $0 \%$ vs. $0.9 \% ; P$ $>0.99$ ), and postoperative bleeding $(2.4 \%$ vs. $1.8 \% ; P=$ $0.56)$. Thirty-eight pairs were matched using propensity score, and the median dissection speed $\left(12 \mathrm{vs} .7 .7 \mathrm{~cm}^{2} / \mathrm{h}\right.$; $P=0.0095)$ was significantly faster in the SSL than in the non-SSL group.

Conclusions ESD for SSL was safely performed, and SSL was smoother to remove than non-SSL. ESD might be an acceptable endoscopic treatment option for SSL.

\section{Introduction}

Sessile serrated lesions (SSLs) are the major precursor lesions of serrated pathway cancers and account for $20 \%$ to $30 \%$ of all sporadic cases of colorectal cancer [1,2]. Despite their importance, they are easily overlooked because they are difficult to detect $[3,4]$. Furthermore, there is a need for not only the diagnosis but an appropriate endoscopic treatment method for SSL.

Previous studies on snare endoscopic mucosal resection (EMR) for colonic polyps $\geq 20 \mathrm{~mm}$ showed that there were significantly more local recurrences in the piecemeal resection
( $11 \%$ to $18 \%$ ) than in the en bloc resection ( $2 \%$ to $3 \%$ ) [5-7]. In addition, in a previous large study on 5 - to $20-\mathrm{mm}$ colonic EMR, incomplete resection was more probable in piecemeal resection compared to en bloc resection ( $20.4 \%$ vs. $8.4 \%$; relative risk 1.41), and was more likely in lesions with 15 - to 20 -mm size compared to 5 - to 7 - $\mathrm{mm}$ size $(23.3 \%$ vs. $5.3 \%$; relative risk 3.21 ) [8].

As Agarwal et al. reported, in an en bloc EMR, the residual lesions were significantly higher at the resection margins in the SSL than in the adenoma group [9]. However, Klein et al. [10] reported the efficacy of thermal ablation of the post-EMR 
mucosal defect margin in reducing polyp recurrence. In another report, cold piecemeal EMR was shown to treat large SSLs adequately, safely, and effectively [11]. Furthermore, endoscopic resection using a snare is also gaining progress in Western countries.

On the other hand, endoscopic submucosal dissection (ESD) for superficial colorectal tumors is technically more difficult and results in a higher risk of adverse events compared to EMR. However, colorectal ESD is currently recognized as a standard technique for treating colorectal tumors because of the development of improved modalities and specialized devices [12]. A number of studies have reported the clinical outcomes of ESD [7], its efficacy for en bloc resection, and the long-term low recurrence rates for large superficial colorectal tumors $[13,14]$.

There have been several studies on the choice of EMR for treating SSL; however, few studies have reported successful use of ESD for SSL. Therefore, its efficacy in ESD for SSL is still unknown. We hypothesized that ESD for SSL may be acceptable if it were as safe and effective as ESD for conventional adenoma. In this study, we examined the characteristics and outcomes of SSL and compared them with those of non-SSL in ESD.

\section{Patients and methods}

\section{Patients}

We retrospectively reviewed patients who were treated between January 2016 and March 2020 at our hospital. Indications for treatment were confirmed using colonoscopy with magnifying function (CF-HQ290ZI or CF-H260AZI; Olympus, Tokyo, Japan). This was determined endoscopically in combination with pit pattern diagnosis using magnifying chromoendoscopy or the Japan NBI Expert Team (JNET) classification by narrow band imaging (NBI) [15-17]. Endoscopic resection was planned for lesions diagnosed as serrated lesion or adenoma, intramucosal carcinoma, submucosal slightly invasive carcinoma $(<1000 \mu \mathrm{m})$. The indications for ESD were as follows: 1$)$ lesions that were difficult to resect with a snare, and that required endoscopic en bloc resection for accurate histological diagnosis, regardless of size (e.g., large depressed-type tumors) [18]; and 2) lesions for which complete resection using snare EMR was difficult. Although SSL is considered to have a lower malignant potential than that of adenomatous lesions, the indication for ESD was decided as above.

We performed colorectal ESD on 370 consecutive cases in 322 patients during the study period. We excluded 390 -Is lesions classified according to the Paris classification [19] from the analysis. The 0 -I lesions of SSL are usually treated with EMR at our center, and all 0-I lesions that underwent ESD were adenoma/intramucosal carcinoma cases. The 0 -I lesions of adenoma/intramucosal carcinoma often had severe fibrosis, and we considered that the dissection was difficult. Therefore, we excluded 0 -I lesions from this study. In addition, we excluded four traditional serrated adenomas, 33 submucosal invasive carcinomas ( 22 submucosal slight invasions and 11 submucosal deeper invasions), 20 submucosal tumors, four local recurrence lesions, one lesion $<10 \mathrm{~mm}$ (lesions $<10 \mathrm{~mm}$ are usually treated
Colorectal ESD : 370

ESD performed for colorectal lesions between January 2016 and March 2020

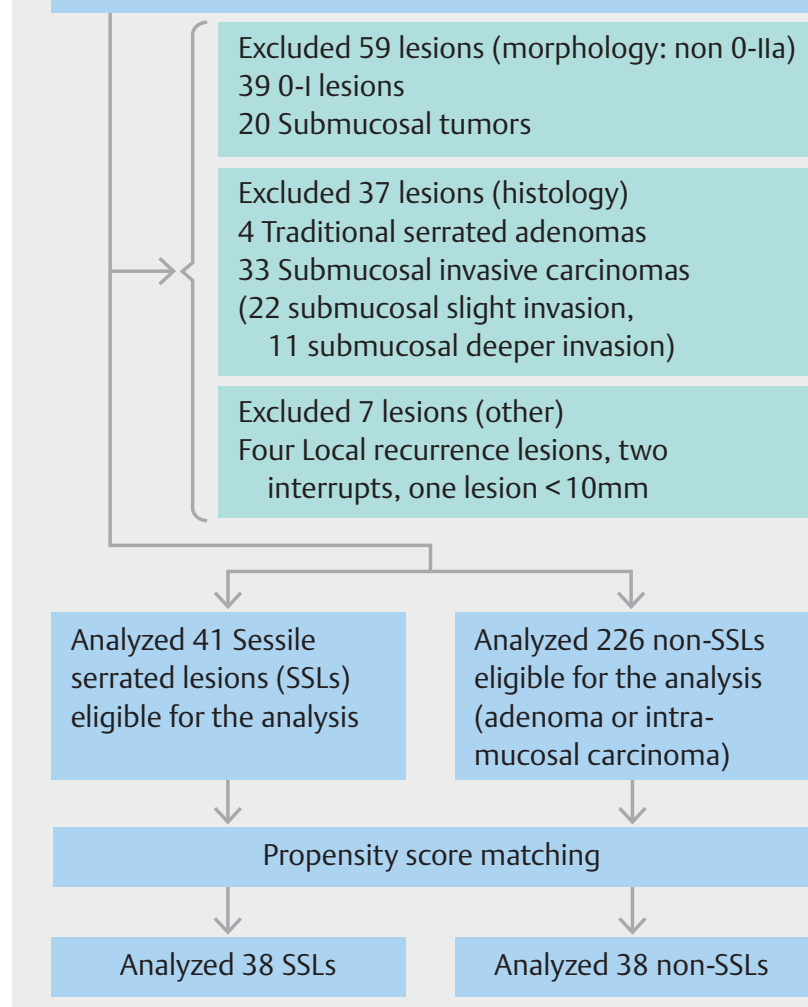

Fig. 1 Study flow diagram. ESD, endoscopic submucosal dissection.

using cold snare polypectomy in our center; this lesion was simultaneously resected with multiple others), and two interrupted lesions (one severe fibrosis and one muscular propria invasion) from the analysis. Finally, 267 0-lla lesions were included. Among these, 41 SSL cases (SSL group) and 226 adenoma/intramucosal carcinoma cases (non-SSL group) ( $>$ Fig. 1) were compared. Almost all intramucosal carcinomas detected arose from adenomas; however, one of the cases was determined to arise from an SSL and was included in the SSL group. Further, 16 SSLs, 19 adenomas, and 12 intramucosal carcinomas were simultaneously resected with multiple lesions. This study was approved by our institutional review board (F2020C34; 8 June 2020). The need for informed consent was waived via the optout method on the hospital's website.

\section{Endoscopic system and ESD procedure}

We used an endoscope with a water-jet system (PCF-Q260]; Olympus). When scope operability was poor because of the paradoxical movement or adhesion, a balloon-assisted endoscope with a hydrophilic-coated silicone splinting tube (ST-CB 1; Olympus) was used [20]. A short-type small-caliber-tip transparent hood (Fujifilm; Tokyo, Japan) was placed on the endoscopic tip. For all the cases, we used carbon dioxide for in- 
sufflation, and the electrosurgical unit was ESG-100 (Olympus). We used a dual knife (KD650Q; Olympus), and ESD was performed under conscious sedation, using intravenous midazolam $(1-3 \mathrm{mg})$, pethidine hydrochloride $(35 \mathrm{mg})$, or both. Submucosal injection of hyaluronic acid solution mixed with a small amount of indigo carmine and $0.1 \%$ epinephrine $(1 \mathrm{~mL}$ of $0.1 \%$ epinephrine in $9 \mathrm{~mL}$ of indigo carmine solution= $1: 100,000$ injectate) was applied. After injection into the submucosal layer, we alternately performed the partial circumferential incision ("Pulse cut slow" mode [30 W]) and the subsequent submucosal dissection ("Forced coagulation" mode [30 W]). We performed all the ESD procedures as described in the previous study [20].

\section{Histological assessment}

Each resected specimen was immersed in $10 \%$ formalin, and a pathologist performed the histological assessment. The histological diagnosis of the lesion and the involvement of the resection margin were evaluated according to the Japanese Classification of Colorectal Carcinoma [21]. The diagnostic category of "intramucosal carcinoma" in Japan is equivalent to "high-grade dysplasia" in Western countries. Sessile serrated lesions were classified as SSL without cytological dysplasia, SSL with cytological dysplasia, traditional serrated adenomas, and hyperplastic polyps, in accordance with the classification provided by the World Health Organization [22]. Histological diagnosis of the nature of intramucosal carcinoma depended on whether there was concomitant detection of tumor belonging to one of the two categories in the adjacent tissue.

\section{Measured characteristics and outcomes}

We drew comparisons between the groups using the following variables: sex and age, tumor size and location, macroscopic type, operator, histology, procedure duration, dissection speed, degree of fibrosis, en bloc resection rate, R0 resection rate, and associated complications. The proximal colon was defined as the cecum, ascending colon, and transverse colon, and the distal colon as the splenic flexure, descending colon, sigmoid colon, and rectum. The 0 -lla lesion was categorized into laterally spreading tumor, granular type (LST-G), and non-granular type (LST-NG) [16]. Considering the operator, Expert (two endoscopists) was defined as having experienced with more than 100 cases of colorectal ESD, and Non-expert (six endoscopists) had experience with fewer than 50 cases of colorectal ESD. There were no endoscopists who had performed between 50 and 100 colorectal ESDs. We further analyzed the fibrosis grade (F0-1: absence, F2-3: presence) [23]. R0 resection was defined as an en bloc resection with free vertical and horizontal margins. $\mathrm{R} 1$ resection (positive resection margin) and $\mathrm{RX}$ resection (unclear/indeterminate resection margin) were included in the category of non-R0 resection. Perforation was considered as being intraoperative, delayed, or both. Postoperative bleeding was referred to as overt bleeding within 14 days after ER, resulting in the need for endoscopic hemostasis.

\section{Statistical analysis}

Group differences were evaluated using the Mann-Whitney $U$ test for the continuous variables and the $X^{2}$ test was used for the dichotomous variables. $P<0.05$ was considered statistically significant. Four factors were considered potential clinical variables for outcomes in ESD: tumor size, location (proximal/ distal), macroscopic type (LST-G/ LST-NG), and operator (Expert/ Non-expert). The propensity score was estimated using a logistic regression model for cases in which we performed ESD for SSL. We performed a one-to-one matching analysis between the SSL and non-SSL groups using the nearest neighbor method, with a caliper width equal to 0.2 of the standard deviation of the logit of the propensity score. The C-statistic $(0.876)$ for evaluating the goodness of fit was calculated. All statistical analyses were performed using EZR (Saitama Medical Center, Jichi Medical University, Saitama, Japan), which is a graphical user interface for R (The R Foundation for Statistical Computing, Vienna, Austria). This is a modified version of the R-commander, which is designed to add statistical functions frequently used in biostatistics [24].

\section{Results}

\section{Baseline characteristics and outcomes}

Baseline characteristics of the ESD patients are presented in - Table 1. Patients in the SSL group were younger than those in the non-SSL group (median age, 58 vs. 71 years; $P<0.001$ ). The percentages of females and proximal colon involvement in the SSL group were significantly higher than those in the nonSSL group (63.4 vs. $35.4 \%$; $P<0.001$ for females and 87.8 vs. $57.1 \%$; $P<0.001$ for proximal colon location). There were four and six cases with lesions involving the appendiceal orifice in the SSL and the non-SSL groups, respectively. There were no significant differences in the median tumor size ( $25 \mathrm{vs}$. $25 \mathrm{~mm} ; P=$ 0.31 ) and percent expert operators ( 44 vs. $47 \%$; $P=0.73$ ) between the groups. In terms of macroscopic type, the rate of LST-NG was significantly higher in the SSL than that in the nonSSL group (97.6 vs. $40.3 \%$; $P<0.001$ ).

Histological results in the SSL group included 39 SSL without cytological dysplasia adenomas, one SSL with cytological dysplasia, and one SSL with carcinoma. The results of the non-SSL group included 113 adenomas and 113 intramucosal carcinomas. Baseline outcomes in the ESD patients are presented in - Table 2. The median duration of the ESD procedure tended to be slightly shorter in the SSL than in the non-SSL group (52 vs. 55 minutes; $P=0.076)$. There were no significant differences between the groups in terms of median dissection speed (12 vs. $\left.11.2 \mathrm{~cm}^{2} / \mathrm{h} ; P=0.44\right)$, presence of fibrosis (2.4 vs. $6.6 \% ; P=$ $0.48)$, en-bloc resection rate (97.6 vs. $99.6 \% ; P=0.74)$, R0 resection rate ( 92.7 vs. $93.4 \% ; P=0.74)$, rate of perforation ( 0 vs. $0.9 \% ; P>0.99)$, and rate of postoperative bleeding (2.4 vs. $1.8 \% ; P=0.56)$. In all cases of non-R0 resection, we could not clearly determine the horizontal margin. There was no perforation in the SSL group. The two cases of perforation in the nonSSL group were conservatively treated after performing endoscopic closure using endoclips. Postoperative bleeding in both 
- Table 1 Clinical characteristics of ESD.

\begin{tabular}{|c|c|c|c|c|c|c|}
\hline & \multicolumn{3}{|c|}{ Baseline cases } & \multicolumn{3}{|c|}{ Propensity-matched cases } \\
\hline & SSL & non-SSL & $P$ & SSL & non-SSL & $P$ \\
\hline & $N=41$ & $N=226$ & & $N=38$ & $\mathrm{~N}=38$ & \\
\hline Sex (female) & $26(63.4)$ & $80(35.4)$ & $<0.001^{1}$ & $23(60.5)$ & $11(28.9)$ & $0.011^{1}$ \\
\hline Age (years); median (range) & $58(38-78)$ & $71(31-88)$ & $<0.001^{2}$ & $58(38-76)$ & $69.5(31-88)$ & $<0.001^{2}$ \\
\hline Location & & & $<0.001^{1}$ & & & $>0.99^{1}$ \\
\hline Proximal colon (\%) & $36(87.8)$ & $129(57.1)$ & & $33(86.8)$ & $33(86.8)$ & \\
\hline Distal colon (\%) & $5(12.2)$ & $97(42.9)$ & & $5(13.2)$ & $5(13.2)$ & \\
\hline Tumor size, median mm (range) & $25(10-50)$ & $25(10-105)$ & $0.31^{1}$ & $25(10-40)$ & $23.5(12-40)$ & $0.61^{\dagger}$ \\
\hline Macroscopic type(LST-G/LST-NG) & $1 / 40$ & $135 / 91$ & $<0.001^{1}$ & $1 / 37$ & $1 / 37$ & $>0.99^{1}$ \\
\hline Operator (Expert/Non-expert) & $18 / 23$ & $106 / 120$ & $0.73^{1}$ & $15 / 23$ & $17 / 21$ & $0.82^{1}$ \\
\hline \multicolumn{7}{|l|}{ Histology } \\
\hline SSL without cytological dysplasia & $39(95.2)$ & & & $37(97.4)$ & & \\
\hline SSL with cytological dysplasia & $1(2.4)$ & & & $1(2.6)$ & & \\
\hline SSL with carcinoma & $1(2.4)$ & & & & & \\
\hline Adenoma & & $113(50)$ & & & $20(52.6)$ & \\
\hline Intramucosal cancer & & $113(50)$ & & & $18(47.4)$ & \\
\hline Fibrosis (presence) (\%) & $1(2.4)$ & $15(6.6)$ & $0.48^{1}$ & $1(2.6)$ & $3(7.9)$ & $0.61^{1}$ \\
\hline \multicolumn{7}{|c|}{$\begin{array}{l}\text { ESD, endoscopic submucosal dissection; SSL, Sessile serrated lesion; non-SSL, adenoma, or intramucosal cancer; Proximal colon (i. e., cecum, ascending colon, and } \\
\text { transverse colon); Distal colon (i. e., splenic flexure, descending colon, sigmoid colon, rectum); LST-G, laterally spreading tumor (granular type); LST-NG, laterally } \\
\text { spreading tumor (non-granular type); Expert, } \geq 100 \text { cases of experience in colorectal ESD, Non-expert, <50 cases of experience in colorectal ESD; Fibrosis, F0-1: } \\
\text { absence/F2-3: presence } \\
{ }^{1} \text { Chi-square test } \\
{ }^{2} \text { Mann-Whitney } U \text { test }\end{array}$} \\
\hline
\end{tabular}

- Table 2 Clinical outcomes of ESD.

\begin{tabular}{|c|c|c|c|c|c|c|}
\hline & \multicolumn{3}{|c|}{ Baseline cases } & \multicolumn{3}{|c|}{ Propensity-matched cases } \\
\hline & SSL & non-SSL & $P$ & SSL & non-SSL & $P$ \\
\hline & $N=41$ & $N=226$ & & $N=38$ & $N=38$ & \\
\hline En bloc resection rate (\%) & $40(97.6)$ & $225(99.6)$ & $0.28^{1}$ & $37(97.4)$ & $37(97.4)$ & $>0.99^{1}$ \\
\hline R0 resection rate $(\%)$ & $38(92.7)$ & $211(93.4)$ & $0.74^{1}$ & $36(94.7)$ & $35(92.1)$ & $>0.99^{1}$ \\
\hline $\begin{array}{l}\text { Duration of ESD procedure (median; min) } \\
\text { (range) }\end{array}$ & $52(5-160)$ & $55(6-300)$ & $0.076^{2}$ & $52.5(5-160)$ & $55(10-210)$ & $0.17^{2}$ \\
\hline Dissection speed (median; $\mathrm{cm}^{2} / \mathrm{h}$ ) (range) & $12(2.8-48)$ & $11.2(1.1-43)$ & $0.44^{2}$ & $12(2.9-48)$ & $7.7(2.7-25)$ & $0.0095^{2}$ \\
\hline Perforation rate (\%) & $0(0)$ & $2(0.9)$ & $>0.99^{1}$ & $0(0)$ & $1(2.6)$ & $>0.99^{1}$ \\
\hline Postoperative bleeding rate (\%) & $1(2.4)$ & $4(1.8)$ & $0.56^{1}$ & $0(0)$ & $1(2.6)$ & $>0.99^{1}$ \\
\hline $\begin{array}{l}\text { ESD, endoscopic submucosal dissection; SSL, se } \\
\text { horizontal margins } \\
{ }^{1} \text { Chi-square test } \\
{ }^{2} \text { Mann-Whitney U test }\end{array}$ & serrated lesion; ! & -SSL, adenoma, or & amucosal c & r; R0 resection, en & loc resection wit & ertical and \\
\hline
\end{tabular}


groups could be managed using endoclips. We successfully managed all adverse events endoscopically.

Of the 41 cases in the SSL group, one lesion was not resected en bloc because of involvement of the appendiceal orifice with severe fibrosis and was divided into two parts with a snare. Similarly, of the 226 cases in the non-SSL group, one lesion could not be resected en bloc because of the involvement of the ileocecal valve and was divided into two parts with a snare. Histological evaluation of the non-en bloc resection cases revealed a positive horizontal margin and a negative vertical margin.

\section{Characteristics and outcomes after propensity score matching}

The matched four factors and the characteristics between the SSL and non-SSL groups after the propensity score matching are shown in > Table 1 . Finally, 38 pairs were matched in the study, and the differences in outcomes of the ESD treatment were compared between the two groups ( $\triangleright$ Table 2 ). Similar to the baseline results, there were statistically significant differences in age $(P<0.001)$ and sex $(P=0.011)$ between the groups. The rate of en bloc resection (97.4\%) was similar in both groups. There were no significant differences in the existence of fibrosis (2.6 vs. $7.9 \% ; P=0.61$ ), the rate of R0 resection (94.7 vs. $92.1 \% ; P>0.99$ ), and adverse events between the groups. The median ESD procedure duration was not significantly different between the groups (52.5 vs. 55 minutes; $P=$ 0.17 ); however, the median dissection speed (12 vs. $7.7 \mathrm{~cm}^{2} / \mathrm{h}$; $P=0.0095)$ was significantly faster in the SSL than in the nonSSL group.

\section{Discussion}

Several studies described the efficacy of ESD for superficial colorectal neoplasia for large lesions, and rates of en bloc and complete resections were higher in ESD than in $\operatorname{EMR}[7,12]$. Rates of en bloc resection (99\%) and R0 resection (93\%) reflected favorable outcomes in baseline cases in this study.

As Agarwal et al. reported, in en bloc resection EMR, incidence of residual lesions at the resection margin was significantly higher in the SSL compared to the adenoma group [9]. This suggests that it may have been more difficult to recognize the border in the SSL than in the adenoma group. In the present study, in the baseline cases, there was no difference in the rates of R0 resection between both groups (SSL $92.7 \%$ vs. non-SSL $93.4 \% ; P=0.74$ ), which was a favorable result. In large lesions with excessive folds, it may be more difficult to identify the border. Some SSLs were covered with a mucus cap. A mucus cap is effective in detecting SSL and it assists in the identification of the borderline. However, a mucus cap may shift from the lesion due to contact during endoscopic resection which may affect the identification of SSLs. In such cases, to avoid misidentifying the borderline, mucosal incision under direct vision in ESD was beneficial. Marking of the incision is not usually necessary in the non-SSL group because the border between the tumor and the normal tissues is quite clear after spraying with indigo carmine. However, SSL borders are usually inconspicuous and when they remain unclear on chromoendoscopy, marking of the borders prior to incision can be useful ( $>$ Fig. 2). After propensity score matching, there were no differences in rates of en bloc and R0 resections between the SSL and the non-SSL groups.

The median dissection speed ( 12 vs. $7.7 \mathrm{~cm}^{2} / \mathrm{h} ; P=0.0095$ ) was significantly faster in the SSL than in the non-SSL group. Only one SSL with submucosal fibrosis was observed in this study. We believe that the absence of fibrosis in SSL was one of the reasons for faster dissection speed. Similarly, Pellise et al. described that SSL was lifted easily after injection and was not associated with submucosal fibrosis [6].

Although dissection speed was significantly higher in the SSL group, the tumor size and procedure time were not different between the two groups. We believe this is because the SSL group had larger horizontal margins than did the non-SSL group, possibly because we marked the borders further outside the lesions in the SSL group. The dissection speed for the nonSSL group after the propensity score matching $\left(7.7 \mathrm{~cm}^{2} / \mathrm{h}\right)$ was lower than that before matching $\left(11.2 \mathrm{~cm}^{2} / \mathrm{h}\right)$. This is probably because the number of LST-Gs decreased after matching. In this study, SSL was significantly more common in younger patients, females, and proximal colon location, which was consistent with the findings of previous reports $[6,25]$. The areas of the proximal colon, especially the ascending colon, have rich fat in the colonic submucosa, and endoscopists must be careful to identify the dissection line in ESD. After a clear submucosal layer under the fat tissue is directly observed, submucosal dissection is relatively easy.

In the SSL group, the only non-en bloc resection was a lesion covering the appendiceal orifice. Jacob et al. reported that the area near the appendiceal orifice was often fibrotic, which increased the difficulty of ESD [26]; the case in the present study had fibrosis of similar severity. SSLs can commonly occur in the appendix [27]; therefore, care must be taken. The traction system may be effective in the ESD lesion involving the appendiceal orifice [28].

Moss et al. reported that in large lesions ( $\geq 20 \mathrm{~mm}$ ), if the initial EMR was deemed successful, the recurrent lesion was usually unifocal and diminutive, and could be managed. Overall, local recurrence was managed endoscopically in $93 \%$ of the cases [5]. Large residual tumors and local recurrences caused by the incomplete resections are very difficult to resect with additional endoscopic treatment; therefore, should be avoided [29]. Moreover, patients with piecemeal resection must undergo intensive endoscopic follow-up, highlighting the importance of en bloc resection. However, in endoscopic treatment for large SSLs, Klein et al. [10] reported a 4-fold reduction in adenoma recurrence after systematic ablation of the postEMR mucosal defect margins. Tutticci et al. reported that the cold piecemeal EMR adequately treats large SSLs with low local recurrence and low postoperative bleeding [11]. Furthermore, endoscopic resection using a snare is widely acceptable in Western countries. However, there is a scope for consideration of ESD in the future. Both ESD and EMR have their advantages and disadvantages, and it is difficult to decide which is useful at this stage. In addition, it is necessary to consider their financial burden. Some studies have been conducted on EMR; nonetheless, no study has demonstrated the indication of ESD in SSL. 

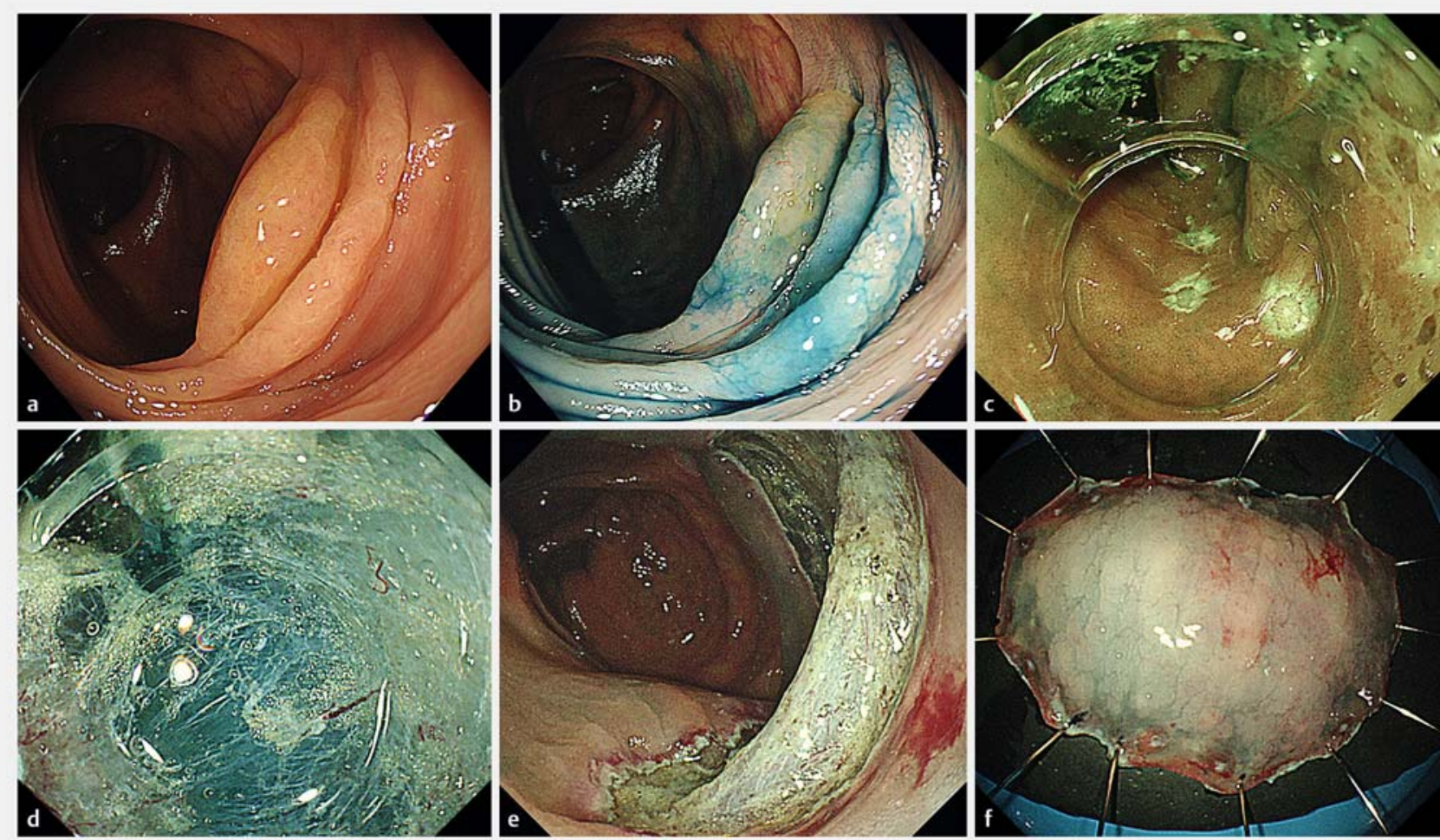

Fig. 2 Sessile serrated lesion at the ascending colon. a Conventional image by white light. b Indigo carmine dye $(0.4 \%)$ was sprayed over the lesion, and the borderline was partially unclear. c A marking under NBI mode was useful for incision. $\mathbf{d}$ Submucosal dissection using a dual knife. More fatty tissue in the submucosa was observed in some cases at deep parts of the colon. e Ulcer bed after en bloc resection. $\mathbf{f}$ Resected specimen. The tumor measured $40 \times 25 \mathrm{~mm}$, and histological evaluation revealed a negative-margin sessile serrated lesion without cytological dysplasia.

It is difficult to determine the indication of ESD for SSL depending on only the lesion size. Although ESD has higher rates of en bloc and complete resection than does EMR, it requires experience and long procedure times. ESD is not required in all cases. Alternatively, a large EMR study that categorized lesions $(\geq 20 \mathrm{~mm})$ by size demonstrated a statistically significant eight-fold increase in the risk of recurrence for adenomas compared with the SSLs in $20-25 \mathrm{~mm}$ lesions. This persisted after adjusting for the potential confounding variables (adjusted hazard ratio 7.6; $P=0.047$ ); nevertheless, this was not maintained in the larger lesions [6]. These findings suggest that $S S L \geq 25 \mathrm{~mm}$ may be good indications for ESD. Further appropriate indications of ESD for large SSLs should be identified and validated using randomized trials and multicenter studies, or with a direct comparison of EMR and ESD.

It is difficult to achieve complete resection using the snare technique in large lesions that exceed the folds and those that involve the appendiceal orifice or the ileocecal valve as with adenomas. Such SSLs may be good indications for ESD because it is difficult to detect the borderline characteristics of SSL.

Considering lesions (e.g., lesions that require accurate histological diagnosis such as an SSL with carcinoma) requiring endoscopic en bloc resection for which the use of the snare technique is difficult, ESD should be listed as an option for endoscopic treatment. The skill and knowledge of an accurate preoperative endoscopic diagnosis for dysplasia or cancer within SSL is indispensable [30-32].

As reported by Byeon et al. [33], the rate of en bloc resection was higher for ESD than for hybrid ESD (snaring resection technique during the ESD procedure with submucosal dissection) ( 87 vs. $64 \%, P<.01$ ). The rate of the histologically complete resection was also higher for ESD. However, the rate of resection of lesions less than $20 \mathrm{~mm}$ was not different between the ESD and the hybrid ESD groups; further, the objects of this study were colorectal adenomatous lesions. According to another report, hybrid ESD for SSL was easy [34]; therefore, in the case of SSL of $20 \mathrm{~mm}$ and under, hybrid ESD may be considered.

Complete removal of SSL may ultimately prevent interval colorectal cancer. However, Yang et al. reported that there was no decrease in the frequency of right-sided colorectal cancer as might be expected with improved recognition and management of predominantly right-sided SSLs [35]. They have discussed the possibility that the diagnostic ability of SSLs or interval in the SSLs-inducing colorectal cancer occurrence was not appropriate in this study. Further validation will require future large-scale, long-term studies.

There are several limitations to our study. First, it was a retrospective cohort study performed at a single institution, and the number of SSLs was small. Second, this study did not consider local recurrence during follow-up colonoscopy. This study 
was based on previous studies that demonstrated that en bloc resection in ESD was associated with less local recurrence. Differentiation of hyperplastic polyps from SSLs was not possible because of the limitations of the JNET classification. Use of NBI classifications that differentiate between serrated lesions and hyperplastic polyps was not routine in our center during the study period. The macroscopic type (e.g., Paris classification, LST sub-type) was a subjective judgment.

\section{Conclusion}

In conclusion, ESD for SSL was safely performed and the tumors were more easily removed than with use of ESD for non-SSL. ESD might be an acceptable endoscopic treatment option for SSL that may be difficult to completely resect even with piecemeal resection by EMR using a snare, or for SSL judged, during preoperative assessment, to require en bloc resection.

\section{Competing interests}

The authors declare that they have no conflict of interest.

\section{References}

[1] Jass JR. Classification of colorectal cancer based on correlation of clinical, morphological and molecular features. Histopathology 2007; 50: $113-130$

[2] Snover DC. Update on the serrated pathway to colorectal carcinoma. Hum Pathol 2011; 42: 1-10

[3] de Wijkerslooth TR, Stoop EM, Bossuyt PM et al. Differences in proximal serrated polyp detection among endoscopists are associated with variability in withdrawal time. Gastrointest Endosc 2013; 77: 617-623

[4] Payne SR, Church TR, Wandell M et al. Endoscopic detection of proximal serrated lesions and pathologic identification of sessile serrated adenomas/polyps vary on the basis of center. Clin Gastroenterol Hepatol 2014; 12: 1119-1126

[5] Moss A, Williams S], Hourigan LF et al. Long-term adenoma recurrence following wide-field endoscopic mucosal resection (WF-EMR) for advanced colonic mucosal neoplasia is infrequent: results and risk factors in 1000 cases from the Australian Colonic EMR (ACE) study. Gut 2015; 64: 57-65

[6] Pellise M, Burgess NG, Tutticci N et al. Endoscopic mucosal resection for large serrated lesions in comparison with adenomas: a prospective multicentre study of 2000 lesions. Gut 2017; 66: 644-653

[7] Oka S, Tanaka S, Saito Y et al. Local recurrence after endoscopic resection for large colorectal neoplasia: a multicenter prospective study in Japan. Am J Gastroenterol 2015; 110: 697-707

[8] Pohl H, Srivastava A, Bensen SP et al. Incomplete polyp resection during colonoscopy-results of the complete adenoma resection (CARE) study. Gastroenterology 2013; 144: 74-80

[9] Agarwal A, Garimall S, Scott Fl et al. En bloc endoscopic mucosal resection is equally effective for sessile serrated polyps and conventional adenomas. Surg Endosc 2018; 32: 1871-1878

[10] Klein A, Tate DJ, Jayasekeran V et al. Thermal ablation of mucosal defect margins reduces adenoma recurrence after colonic endoscopic mucosal resection. Gastroenterology 2019; 156: 604-613

[11] Tutticci NJ, Hewett DG. Cold EMR of large sessile serrated polyps at colonoscopy (with video). Gastrointest Endosc 2018; 87: 837-842
[12] Saito Y, Yamada M, So E et al. Colorectal endoscopic submucosal dissection: technical advantages compared to endoscopic mucosal resection and minimally invasive surgery. Dig Endosc 2014; 26: 52-61

[13] Yamada M, Saito Y, Takamaru $\mathrm{H}$ et al. Long-term clinical outcomes of endoscopic submucosal dissection for colorectal neoplasms in 423 cases: a retrospective study. Endoscopy 2017; 49: 233-242

[14] Shigita K, Oka S, Tanaka S et al. Long-term outcomes after endoscopic submucosal dissection for superficial colorectal tumors. Gastrointest Endosc 2017; 85: 546-553

[15] Sano Y, Tanaka S, Kudo SE et al. Narrow-band imaging (NBI) magnifying endoscopic classification of colorectal tumors proposed by the Japan NBI Expert Team. Dig Endosc 2016; 28: 526-533

[16] Kudo S, Lambert R, Allen Jl et al. Nonpolypoid neoplastic lesions of the colorectal mucosa. Gastrointest Endosc 2008; 68: S3-47

[17] Uraoka T, Saito Y, Matsuda T et al. Endoscopic indications for endoscopic mucosal resection of laterally spreading tumours in the colorectum. Gut 2006; 55: 1592-1597

[18] Tanaka S, Kashida H, Saito Y et al. JGES guidelines for colorectal endoscopic submucosal dissection/endoscopic mucosal resection. Dig Endosc 2015; 27: 417-434

[19] The Paris endoscopic classification of superficial neoplastic lesions: esophagus, stomach, and colon: November 30 to December 1, 2002. Gastrointest Endosc 2003; 58: S3-43

[20] Kuroki Y, Asonuma K, Uehara $\mathrm{N}$ et al. Efficacy and suitable indication of colorectal endoscopic submucosal dissection using a balloon-assisted endoscope. JGH Open 2020; 4: 185-190

[21] Watanabe T, Itabashi M, Shimada Y et al. Japanese Society for Cancer of the Colon and Rectum (JSCCR) guidelines 2010 for the treatment of colorectal cancer. Int J Clin Oncol 2012; 17: 1-29

[22] Pai RK, Mäkinen M], Rosty C. Colorectal serrated lesions and polyps. WHO Classification of Tumours of the Digestive System, 5th ed. International Agency for Research on Cancer (IARC); 2019: 163-169

[23] Matsumoto A, Tanaka S, Oba S et al. Outcome of endoscopic submucosal dissection for colorectal tumors accompanied by fibrosis. Scand J Gastroenterol 2010; 45: 1329-1337

[24] Kanda Y. Investigation of the freely available easy-to-use software 'EZR' for medical statistics. Bone Marrow Transplant 2013; 48: 452458

[25] Murakami T, Sakamoto N, Ritsuno H et al. Distinct endoscopic characteristics of sessile serrated adenoma/polyp with and without dysplasia/carcinoma. Gastrointest Endosc 2017; 85: 590-600

[26] Jacob H, Toyonaga T, Ohara Y et al. Endoscopic submucosal dissection of cecal lesions in proximity to the appendiceal orifice. Endoscopy 2016; 48: 829-836

[27] Rubio CA. Serrated adenomas of the appendix. J Clin Pathol 2004; 57: 946-949

[28] Oung B, Rivory J, Chabrun E et al. ESD with double clips and rubber band traction of neoplastic lesions developed in the appendiceal orifice is effective and safe. Endosc Int Open 2020; 8: E388-E395

[29] Kuroki Y, Hoteya S, Mitani T et al. Endoscopic submucosal dissection for residual/locally recurrent lesions after endoscopic therapy for colorectal tumors. J Gastroenterol Hepatol 2010; 25: 1747-1753

[30] Burgess NG, Pellise M, Nanda KS et al. Clinical and endoscopic predictors of cytological dysplasia or cancer in a prospective multicentre study of large sessile serrated adenomas/polyps. Gut 2016; 65: 437446

[31] Tanaka Y, Yamano HO, Yamamoto E et al. Endoscopic and molecular characterization of colorectal sessile serrated adenoma/polyps with cytologic dysplasia. Gastrointest Endosc 2017; 86: 1131-1138

[32] Sano W, Fujimori T, Ichikawa K et al. Clinical and endoscopic evaluations of sessile serrated adenoma/polyps with cytological dysplasia. I Gastroenterol Hepatol 2018; 33: 1454-1460 
[33] Byeon JS, Yang DH, Kim KJ et al. Endoscopic submucosal dissection with or without snaring for colorectal neoplasms. Gastrointest Endosc 2011; 74: 1075-1083

[34] Tamaru Y, Kuwai T, Kuroki K et al. Usefulness and safety of colorectal precutting EMR and hybrid endoscopic submucosal dissection for sessile serrated polyps with use of a novel multifunctional snare. VideoGIE 2019; 4: 276-278
[35] Yang JF, Noffsinger AE, Agrawal D et al. Colorectal cancer anatomic distribution patterns remain the same after sessile serrated adenoma/polyp considered cancer precursor: a 9-year comparison study from community-based endoscopy centers. J Gastrointest Oncol 2016; 7: 917-923 